\title{
(6) OPEN ACCESS \\ Intra-abdominal Candida spp infection in acute abdomen in a quality assurance (QA)-certified academic setting
}

\author{
Vito Rodolico, ${ }^{1}$ Paola Di Carlo, ${ }^{2}$ Gaspare Gulotta, ${ }^{3}$ Francesco D'Arpa, ${ }^{3}$ \\ Giuseppe Salamone, ${ }^{3}$ Gianfranco Cocorullo, ${ }^{3}$ Antonino Agrusa, ${ }^{3}$ Anna Giammanco, ${ }^{4}$ \\ Consolato Sergi ${ }^{5,6}$
}

${ }^{1}$ Department of Sciences for Health Promotion and Mother \& Child Care, Section of Anatomic Pathology, University of Palermo, Palermo, Italy 2Department of Sciences for Health Promotion and Mother \& Child Care, Section of Infectious Disease, University of Palermo, Palermo, Italy

${ }^{3}$ Department of General Surgery and Emergency, University of Palermo, Palermo, Italy

${ }^{4}$ Department of Sciences for Health Promotion and Mother \& Child Care, Section of Microbiology, University of Palermo, Palermo, Italy ${ }^{5}$ Department of Lab. Medicine and Pathology, University of Alberta, Edmonton, Alberta, Canada

${ }^{6}$ Stollery Children's Hospital, University of Alberta, Edmonton Alberta, Canada

\section{Correspondence to}

Professor Consolato Sergi, Department of Lab Medicine and Pathology, University of Alberta, Edmonton, Alberta, Canada T6G 2B7;

sergi@ualberta.ca

Received 30 August 2016 Revised 4 November 2016 Accepted 9 November 2016 Published Online First

9 December 2016

\section{ABSTRACT}

Aims To evaluate the contribution of light microscopy to detecting Candida spp infection in patients with complicated intra-abdominal infections (IAls) admitted for acute abdomen to a quality assurance (QA)-certified surgical emergency ward.

Methods We conducted a retrospective study (20082012) of 809 abdominal intraoperative or biopsy tissue specimens obtained from patients admitted with acute abdomen and microbiological samples positive for Candida spp. Demographic data, mortality, comorbidities, specimen type, microscopy results, special histological staining performed, antimicrobial therapy were collected and analysed. Any comments at the multidisciplinary team meeting was recorded in minutes of and approved.

Results Sixty-six patients with complicated IAls due to Candida spp were identified (39 male, 27 female, mean \pm SD age $75 \pm 20$ years). Candida albicans was isolated in 35 cases and Candida non-albicans spp in 31 cases. Candida spp were isolated from blood in $50 \%$ of all selected microbiological specimens. Patients were stratified according to Candida spp (albicans vs nonalbicans), underlying cancer disease and no previous antimicrobial administration, and a positive correlation with C. albicans isolation was found ( $p=0.009$ and $p=0.048$, respectively). Out of 41 cases with microscopic evaluation, we identified yeast forms, pseudohyphae or both, indicative of Candida spp, in 23. Identification of Candida spp in histological specimens was higher in C. albicans cases than in C. non-albicans cases $(73 \%$ vs $37.5 \%$ ). Microscopy allowed prompt treatment of all patients.

Conclusions Light microscopy still has great diagnostic significance, being a solid QA step. It provides rapid information and clues in patients who may harbour impaired defence mechanisms, concurrent chronic conditions and/or cancer.

\section{INTRODUCTION}

Intra-abdominal infections (IAIs) are among the most complicated and life-threatening infections to diagnose and treat in an emergency department. A successful outcome depends inevitably on early diagnosis, rapid and appropriate surgical intervention, and selection of the most appropriate antiinfective treatment. ${ }^{1-3}$ Tissue morphology and culture provide important hints for the management of patients with complicated IAIs. Intra-abdominal candidiasis is a major cause of morbidity in patients undergoing intra-abdominal surgery. It is mainly represented by, but not limited to, Candida peritonitis or intra-abdominal abscesses in patients who have had abdominal surgery. ${ }^{4} 5$ High mortality from Candida peritonitis (38\%) was reported in a prospective survey of 93 patients admitted to the intensive care unit. ${ }^{5}$ Comorbidities, particularly inflammatory bowel disease, may be a challenge. Indeed, ulcerative colitis (UC) is associated with defects in the colonic mucosa barrier and bacterial dysbiosis in association with a reduction in mucous-containing goblet cells and mucous production. In both Crohn's disease patients with ileitis and UC patients with normal histology, bacteria coated with IgA and IgG penetrate the mucin layer as previously identified. ${ }^{6-8}$ Candida spp are the main fungal strains of gut flora, and gastrointestinal tract surgery may lead to mucosal disruption, resulting in Candida spp dissemination into the bloodstream. ${ }^{5}$

Owing to a lower prevalence of culture-proven cases of invasive fungal infections, non-culture-based methods are increasingly used for early diagnosis to improve patient outcome. ${ }^{9}$ New diagnostic algorithmic molecular platforms have high sensitivity and specificity in detecting fungi in clinical specimens. ${ }^{9-11}$ PCR-based assays have indeed improved the target of treatment, but molecular analysis does not help to discriminate 'infection' from 'colonisation'. Currently, Candida spp, once considered harmless, may trigger life-threatening infections in specific critical settings. Despite the availability of PCR-based assays, there is still a lack of satisfactory specificitythat is, prompt distinction between C. albicans infection and 'contaminants' and this distinction is not clear in critically ill surgical patients. ${ }^{12-15}$ Thorough review of the literature reveals that identification of yeasts in sterile samples by microbiological methods provides insight in predicting invasive fungal infections, but these methods remain uncertain in predicting infection or colonisation in non-sterile samples, which is the case with surgical samples. ${ }^{14}{ }^{15}$ Therefore, microbiology should be complemented with cyto- and/or histo-pathology, particularly in patients with intra-abdominal candidiasis. ${ }^{3}{ }^{16-18}$ The value of light microscopic examination has not been undermined by new techniques. Light microscopy is still one of the major diagnostic tools in mycology, because it allows morphological characterisation of fungi. Histological examination allows determination of the amount and morphology of budding hyphae. ${ }^{1920}$ 
Clinical audits are vital in quality assurance (QA)-certified institutions, particularly in emergency settings, and consist of quality processes aiming to improve patient care and outcomes through systematic review of care against explicit standards/criteria with enactment of changes in practice if needed. ${ }^{21} 22$ The aim of this investigation was to evaluate the contribution of histopathology in surgical patients with acute abdomen and complicated IAIs with cultures positive for Candida spp.

\section{MATERIALS AND METHODS}

This is a retrospective study of patients with positive Candida infection identified microbiologically, harbouring complicated IAI, in an external QA-certified general surgery and emergency academic unit between 2008 and 2012. In accordance with the criteria definitions, we included all patients with IAIs identified according to the current guidelines. ${ }^{2} 4{ }^{23}$ Antifungal therapy for patients with severe community-acquired or healthcare-associated (nosocomial) infection is recommended if Candida is grown from intra-abdominal cultures. Our enrolled surgical patients showed at least one of the following specimens positive for Candida spp-peritoneal fluid, peritoneal fluid cultures, drainage fluid/blood or bile or tissue-during surgical procedures according to the Infectious Diseases Society of America and the American Society for Microbiology. ${ }^{10}$ In our institution, the BACTEC 9050 system (Becton Dickinson Diagnostic Instrument System, Paramus, New Jersey, USA) is used as the first step in the culture procedure. ${ }^{24}$ Candida spp were also identified by conventional morphological and biochemical methods as previously reported. ${ }^{24-26}$ A multiplex real-time PCR (LightCycler Septifast, MGRADE; Roche Molecular Diagnostics, Prague, Czech Republic) was used as an additional diagnostic tool for selective detection of five Candida spp (C. albicans, C. parapsilosis, C. tropicalis, C. krusei, C. glabrata). ${ }^{27-32}$ Demographic data, predisposing factors, and clinical, endoscopic and radiological features were collected from the electronic medical records of the patients (figure 1A). 809 concurrent surgical pathology specimens obtained under the categories of tissue, aspirate, abscess or biopsy available for cyto- and/or histo-pathological examination by surgical pathologists were included to measure, compare and evaluate the fungi accurately. The most common emergency surgical procedures requiring morphological examination were: clinical/radiological diagnosis of obstruction and perforating lesions due to malignant tumours $(57 \%)$, peritonitis secondary to perforating lesions (34\%), intra-abdominal abscess (27\%), surgical complications of Crohn's disease (16\%), postoperative complications of biliary surgery, and acute cholecystitis (comorbidity) (12\%). The cyto- and/or histo-pathological examination was performed in a QA-certified hospital setting. All histological slides were reviewed by one pathologist (VR), who were blinded to the clinical and laboratory data. H\&E, Gomori's methenamine silver (GMS) and periodic acid-Schiff (PAS) staining was routinely carried out. Special stains were also used for cytological cases if material stored in the archives was considered satisfactory and adequate. Light microscopic confirmation of yeast forms, pseudohyphae or both and characterisation of Gram-positive additional stains on histological sections were classified according to international standards. ${ }^{23} 33 \quad 34$ The microbiological records were systematically verified for corresponding culture reports. Any comments of the multidisciplinary team meetings was recorded in minutes and approved. The study protocol conformed with the ethics guidelines of the Declaration of Helsinki for clinical studies and was approved by the local university ethics committee (institutional review board).

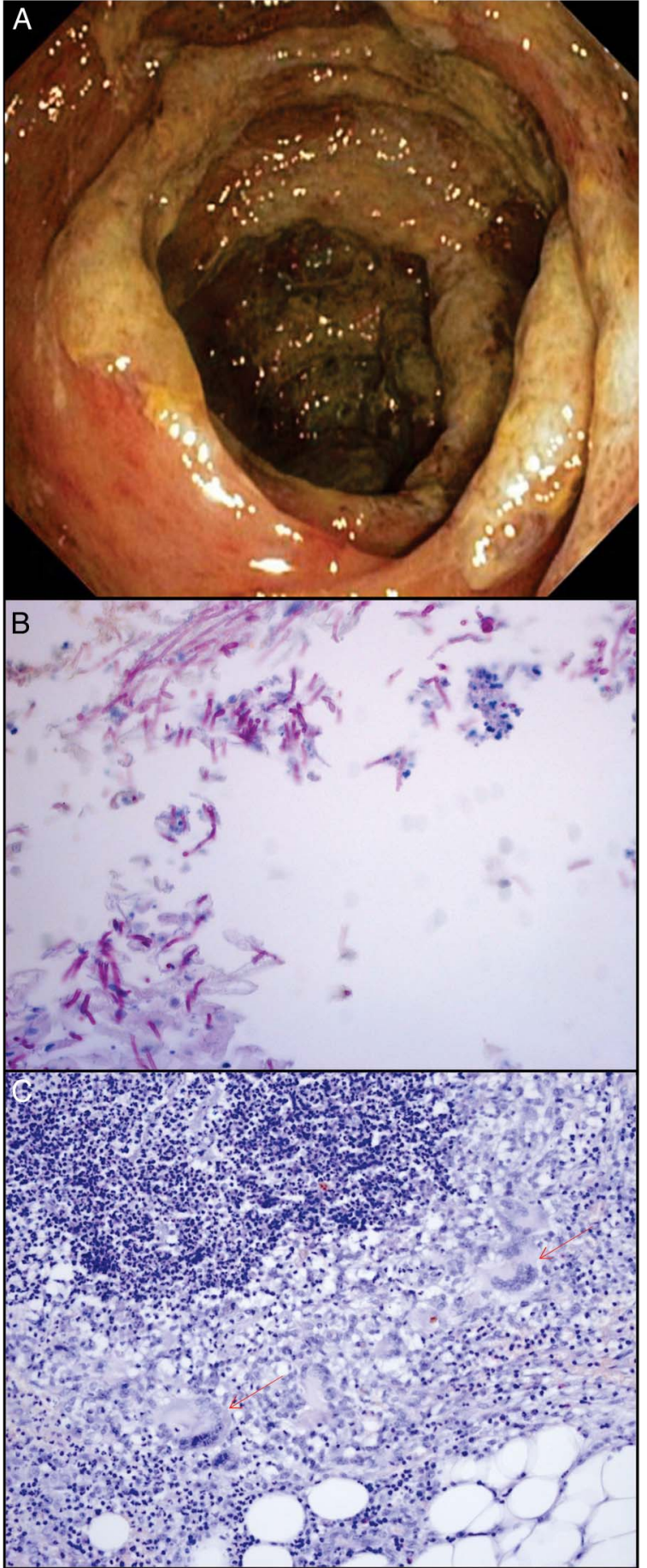

Figure 1 (A) Endoscopic colonic lesions showing ulcers and necrosis. (B) Candida albicans species at the surgical site (periodic acid-Schiff (PAS); $\times 200)$. (C) Chronic inflammatory process with giant cells (arrows), histiocytes and lymphocytes in a patient affected by post-surgical complications due to C. tropicalis and Gemella morbillorum (PAS; $\times 100)$.

\section{Statistical analysis}

Statistical analysis of quantitative and qualitative data, including descriptive statistics, was performed for all items by the biostatics service of our institution. Continuous data are expressed as mean $\pm \mathrm{SD}$, unless otherwise specified. Frequency analysis was performed with the $\chi^{2}$ test or the Fisher exact test, if indicated. ORs and their 95\% CIs were calculated using univariate logistic regression analysis. Data were analysed with Epi Info software 
(V.6.0) and IBM SPSS software (V.21.0). All p values were two sided, and an $\alpha$-level of 0.05 was considered statistically significant. This means that we were willing to accept a probability of $5 \%$ of making a type I error in assuming that a relationship between two variables exists when it really does not.

\section{RESULTS}

We identified 66 cases of complicated IAI with Candida spp isolated from microbiological specimens during a 5 year study period. These were 39 men and 27 women with ages ranging from 20 to 94 years (mean \pm SD $75 \pm 20$ years). The predisposing factors were identified in 58 patients and included cancer (26), diabetes mellitus (22), autoimmune disease (8) and systemic lupus erythematosus (2). Table 1 shows details of the demographic and clinical characteristics of our patients with culture samples positive for Candida spp.

C. albicans was isolated in 35 patients (mean age 73 \pm 20.8 years), and C. non-albicans spp (C. tropicalis 19\%; C. parapsilosis 16\%; C. glabrata 8\%; C. lusitaniae 7.5\%) in 31 patients (mean age $78.4 \pm 18$ years). In $50 \%$ of all selected microbiological specimens, Candida spp were isolated from blood by culture and/or PCR analysis.

Patients were stratified according to the Candida spp (albicans vs non-albicans), underlying cancer disease and no previous antimicrobial administration, and a positive correlation was found with C. albicans isolation (C. albicans, OR 4.53, 95\% CI 1.47 to $13.97 ; \mathrm{p}=0.009 ;$ C. non-albicans, OR $0.199 ; 95 \% \mathrm{CI}$ 0.039 to $0.997 ; p=0.048)$.

The fatality rate was $3.4 \%$, and 41 of the 66 patients $(62.1 \%)$ had specimens available for histology (34) or cytopathology (7). Among these 41 cases, we identified yeast forms, pseudohyphae or both, indicative of Candida spp, in 23, five of which were identified by cytopathological analysis only (figure 1B).

In the remaining 18 histological specimens that were negative for Candida spp, we observed an accompanying chronic

Table 1 Characteristics of 66 enrolled surgical patients

\begin{tabular}{ll}
\hline Age (mean \pm SD), years (range) & $75 \pm 20$ (20-94) \\
Male/female ( $)$ & $39 / 27$ \\
History of abdominal surgery & 39 \\
Samples positive for Candida spp (n) & \\
Peritoneal fluid & 18 \\
Blood smear & 22 \\
Blood PCR & 30 \\
Biopsy/resection & 23 \\
Percutaneous aspiration biopsy of abscess & 40 \\
Bile sample aspirate & 17 \\
Previous surgery & 37 \\
Antibiotics at home & 51 \\
Location and aetiologies of complicated IAI (n) & \\
Lower gastrointestinal tract & \\
Appendicitis & 7 \\
Diverticulitis & 10 \\
Malignancy & 15 \\
Inflammatory bowel disease & 5 \\
Ischaemic & 5 \\
Miscellaneous & 10 \\
Upper intestinal tract & \\
Biliary disease & 8 \\
Malignancy & 6 \\
\hline IAl, intra-abdominal infection. &
\end{tabular}

inflammatory process often with giant cells (figure 1C) and/or perivascular lymphocytic infiltration in 13 histological specimens associated with degenerative necrotic phenomena in places. In five cases, the examination was considered negative, because the histological material was inadequate for evaluation or the cytological samples from abscesses were full of neutrophils. Table 2 shows the results of histological diagnosis based on the morphology of fungal forms and positive cultures. Identification of Candida spp in histological specimens was higher in C. albicans cases than in C. non-albicans cases $(73 \%$ vs $37.5 \%)$. Importantly, the analysis of associated risk factors in 23 patients showed that C. albicans was more often seen in patients with cancer $(p=0.033)$ and those with a history of quinolone treatment $(p=0.036)$ than in other groups. In 14 blood negative patients, the diagnosis of Candida spp infection was confirmed by histopathological examination (table 2). Figure 1C shows a chronic inflammatory process with giant cells in a patient affected with post-surgical intra-abdominal complications due to C. tropicalis and Gemella morbillorum bacteria.

\section{DISCUSSION}

C. albicans is the most common opportunistic fungal pathogen, which switches its morphology from single-cell yeast to filament through various signalling pathways after interaction with diverse local stimuli. In this 5 year retrospective single-centre study, we selected surgical patients with complicated IAI who had at least one microbiological sample positive for Candida spp. This research was followed by cyto-/histo-pathological investigation to evaluate the invasiveness, shape and distribution of yeast(s) with pseudohyphal growth, and a study of the correlation between histology, microbiology laboratory results and risk factors for candidiasis in the setting of a QA programme.

The prevalence of C. albicans and C. non-albicans were comparable. In line with the literature, the prevalence of C. nonalbicans spp is increasing in critically ill surgical patients. ${ }^{35}$ Our patients showed a high prevalence of immunosuppression following cancer, which was the major cause of admission for emergency surgery. Cases of malignancy and the number of older people may indeed contribute to the increased prevalence of C. non-albicans spp. A recent cancer study ${ }^{36}$ similarly showed that C. albicans and other Candida spp had the same

Table 2 Comparative analysis of 23 histological examinations positive for Candida spp and surgical microbiological samples positive for CA and C-NA

\begin{tabular}{|c|c|c|c|c|c|}
\hline $\begin{array}{l}\text { Microbiological } \\
\text { specimen } \\
\text { positive for } \\
\text { Candida spp }\end{array}$ & $\mathbf{n}$ & $\begin{array}{l}\text { Histological } \\
\text { concordance }\end{array}$ & $\begin{array}{l}\text { Candida } \\
\text { species }\end{array}$ & Comorbidity & $\begin{array}{l}\text { Outcome } \\
\text { (Died) }\end{array}$ \\
\hline Blood & 7 & 1 & $\begin{array}{l}C A=4 \\
C-N A=3\end{array}$ & $\begin{array}{l}\text { Cancer=6; } \\
\text { cholecystitis=1 }\end{array}$ & $\begin{array}{l}C A=2 \\
C-N A=1\end{array}$ \\
\hline Bile & 4 & - & $\begin{array}{l}\mathrm{CA}=1 \\
\mathrm{C}-\mathrm{NA}=3\end{array}$ & $\begin{array}{l}\text { Cancer=2 } \\
\text { Chronic } \\
\text { gastritis and } \\
\text { cholecystitis=2 }\end{array}$ & \\
\hline Drainage fluid & 6 & 6 & $\begin{array}{l}\mathrm{CA}=4 \\
\mathrm{C}-\mathrm{NA}=2\end{array}$ & $\begin{array}{l}\text { Cancer }=4 \\
\text { Fistula }=2\end{array}$ & $C A=3$ \\
\hline Biopsy specimen & 4 & 4 & $\begin{array}{l}C A=3 \\
C-N A=1\end{array}$ & $\begin{array}{l}\text { Cancer }=2 \\
\text { Fistula }=2\end{array}$ & $C A=1$ \\
\hline Bile plus blood & 2 & 2 & $C A=2$ & $\begin{array}{l}\text { Cancer }=1 \\
\text { Peritonitis=1 }\end{array}$ & \\
\hline
\end{tabular}


prevalence and that candidaemia was associated with substantial mortality. Antifungal prophylaxis and remission of cancer predicted better survival.

In our study, the role of pathology in supporting clinical microbiology is emphasised, particularly with the careful evaluation of Candida strain isolation from surgical specimens and QA programmes. As infectious clinician consultants in surgical and emergency departments, we find that microbiological identification in non-sterile sites, as often occurs in IAIs, does not help to discriminate between contamination and real infection. In fact, both Enterobacteriaceae and Candida albicans can be the predominant microbial species of flora in the human gut and also a common cause of IAIs. ${ }^{3}{ }^{17} 37$ In our case of co-infection due to C. tropicalis and G. morbillorum bacteria, we observed a chronic inflammatory process with a prevalence of giant cells. In $50 \%$ of our patients, Candida spp were detected in the blood by molecular investigation, and, in half of the studied population, Candida spp were detected in surgical samples, such as drainage fluid or bile aspirate. With regard to the usefulness of histological examination, we found that, in 14 blood negative patients, the diagnosis of Candida infection was confirmed by histopathological examination, which can be considered the gold standard. The importance and suitability of histological examination has been highlighted by international recommendations for the diagnosis of intra-abdominal diseases, and case reports and retrospective QA studies have shown the role of Candida spp in chronic or persistent infection in surgical patients, especially those with biliary or gastric diseases. $^{10} 14153839$ Thus, as endoscopic investigations are not able to discriminate the aetiology of a lesion, histology remains of paramount importance. ${ }^{3} 17$ Furthermore, accurate fungal identification by histopathological examination was not operator dependent, with only minor discordance between pathologists and a $\kappa$ value close to 1 . However, for QA purposes, we occasionally found specimens submitted to the surgical pathologists that were inadequate or not representative of the lesion in question, in which case the patient's course may be jeopardised. Most fungi are easy to identify by H\&E staining alone and/or in combination with a histochemical (special) stain such as PAS and GMS. However, these stains cannot distinguish between morphologically similar fungi with potential differences in susceptibility to antimycotic drugs. Currently, the final identification of fungi relies on culture, but this may take several days or longer to yield a definitive result, and surgical pathology units may not have access to fresh tissue. Close collaboration between mycologists and the emergency department is mandatory. In our study, for 41 of 66 positive microbiological samples, there were specimens available for cyto- or histo-pathological examination suggesting invasiveness. Another interesting aspect is that cancer patients are more prone to harbouring infections caused by C. albicans, and our data are strengthened by recent studies that correlate candidaemia with cancer. ${ }^{36} 40-42$

The use of immunohistochemical and in situ hybridisation analysis may be considered for the diagnosis of C. albicans, although they are more expensive techniques than using $\mathrm{H} \& \mathrm{E}$ and special stains.

In conclusion, histopathology provides insight into the diagnostic significance of Candida spp isolated from surgical specimens other than blood samples and helps to provide a prompt result for emergency department staff, enabling them to select the correct treatment (fluconazole vs echinocandin) and potentially avoid the emergency situation of resistance of fungal species.

\section{Take home messages}

- Intra-abdominal candidiasis is a major cause of morbidity in patients undergoing intra-abdominal surgery.

- In more than half of cases with a microscopic evaluation, yeast forms, pseudohyphae or both, indicative of Candida spp, were evident.

- Light microscopy is still of utmost diagnostic significance, being a solid QA step, and its value has not been undermined by new techniques allowing morphological characterisation of fungi and prompt treatment of patients in intensive care units.

\section{Handling editor Slade Jensen}

Twitter Follow Paola Di Carlo at @paoladicarlo19@gmail.com

Acknowledgements We thank Professor Alessandra Casuccio for providing statistical, methodological and software expertise.

Contributors VR and PDC wrote the first draft of the manuscript. GG, FDA, GS, GC and AA identified patients and settings and provided care and follow-up. VR reviewed the original histological slides. AG provided the microbiological diagnosis. CS reviewed the first draft of the manuscript, critically appraised the study and data, and wrote the final version of the manuscript.

\section{Competing interests None declared.}

Ethics approval University of Palermo.

Provenance and peer review Not commissioned; externally peer reviewed.

Open Access This is an Open Access article distributed in accordance with the Creative Commons Attribution Non Commercial (CC BY-NC 4.0) license, which permits others to distribute, remix, adapt, build upon this work non-commercially, and license their derivative works on different terms, provided the original work is properly cited and the use is non-commercial. See: http://creativecommons.org/ licenses/by-nc/4.0/

\section{REFERENCES}

1 Montravers $\mathrm{P}$, Dupont $\mathrm{H}$, Gauzit R, et al. Candida as a risk factor for mortality in peritonitis. Crit Care Med 2006;34:646-52.

2 Menichetti F, Sganga G. Definition and classification of intra-abdominal infections. J Chemother 2009:21(Suppl 1):3-4.

3 Di Carlo P, Vitale F, O'Súilleabhain C, et al. Management of intra-abdominal infections due to carbapenemase-producing organisms. Curr Infect Dis Rep 2014; 16:428.

4 Bassetti M, Marchetti M, Chakrabarti A, et al. A research agenda on the management of intra-abdominal candidiasis: results from a consensus of multinational experts. Intensive Care Med 2013;39:2092-106.

5 Montravers P, Mira JP, Gangneux JP, et al. AmarCand study group. A multicentre study of antifungal strategies and outcome of Candida spp. peritonitis in intensive-care units. Clin Microbiol Infect 2011;17:1061-7.

6 Alipour M, Lou Y, Zimmerman D, et al. A balanced IL-1 $\beta$ activity is required for host response to Citrobacter rodentium infection. PLOS ONE 2013;8:e80656.

7 Alipour M, Zaidi D, Valcheva R, et al. Mucosal barrier depletion and loss of bacterial diversity are primary abnormalities in paediatric ulcerative colitis. J Crohns Colitis 2016;10:462-71.

8 Liu JJ, Davis EM, Wine E, et al. Epithelial cell extrusion leads to breaches in the intestinal epithelium. Inflamm Bowel Dis 2013;19:912-21.

9 Clancy CJ, Nguyen MH. Finding the "missing 50\%" of invasive candidiasis: how nonculture diagnostics will improve understanding of disease spectrum and transform patient care. Clin Infect Dis 2013;56:1284-92.

10 Baron EJ, Miller JM, Weinstein MP, et al. A guide to utilization of the microbiology laboratory for diagnosis of infectious diseases: 2013 recommendations by the Infectious Diseases Society of America (IDSA) and the American Society for Microbiology (ASM). Clin Infect Dis 2013;57:e22-121.

11 Tissot F, Lamoth F, Hauser PM, et al. $\beta$-glucan antigenemia anticipates diagnosis of blood culture-negative intraabdominal candidiasis. Am J Respir Crit Care Med 2013; 188:1100-9.

12 León C, Ostrosky-Zeichner L, Schuster M. What's new in the clinical and diagnostic management of invasive candidiasis in critically ill patients. Intensive Care Med 2014;40:808-19. 
13 Pittet $\mathrm{D}$, Monod M, Suter PM, et al. Candida colonization and subsequent infections in critically ill surgical patients. Ann Surg 1994;220:751-8.

14 Watts JC. Surgical pathology and the diagnosis of infectious diseases. Am I Clin Pathol 1994:102:711-12.

15 Zhang L, Xiao M, Watts MR, et al. Development of fluconazole resistance in a series of Candida parapsilosis isolates from a persistent candidemia patient with prolonged antifungal therapy. BMC Infect Dis 2015;15:340.

16 Eggimann P, Pittet D. Candida colonization index and subsequent infection in critically ill surgical patients: 20 years later. Intensive Care Med 2014;40:1429-48.

17 Di Carlo P, Di Vita G, Guadagnino G, et al. Surgical pathology and the diagnosis of invasive visceral yeast infection: two case reports and literature review. World J Emerg Surg 2013;8:38.

18 Santos LD, Rogan KA, Kennerson AR. Cytologic diagnosis of suppurative cholecystitis due to Candida albicans and actinomyces. A report of 2 cases. Acta Cytol 2004;48:407-10.

19 Sangoi AR, Rogers WM, Longacre TA, et al. Challenges and pitfalls of morphologic identification of fungal infections in histologic and cytologic specimens: a ten-year retrospective review at a single institution. Am I Clin Pathol 2009;131:364-75.

20 Challa S, Pamidi U, Uppin SG, et al. Diagnostic accuracy of morphologic identification of filamentous fungi in paraffin embedded tissue sections: correlation of histological and culture diagnosis. Indian I Pathol Microbiol 2014;57:583-7.

21 Brown A, Santilli M, Scott B. The internal audit of clinical areas: a pilot of the internal audit methodology in a health service emergency department. Int I Qual Health Care 2015:27:520-2.

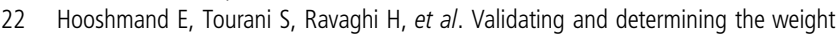
of items used for evaluating clinical governance implementation based on analytic hierarchy process model. Int J Health Policy Manag 2015;4:645-51.

23 Solomkin JS, Mazuski JE, Bradley JS, et al. Diagnosis and management of complicated intra-abdominal infection in adults and children: guidelines by the Surgical Infection Society and the Infectious Diseases Society of America. Clin Infect Dis 2010;50:133-64

24 Cortegiani A, Russotto V, Montalto F, et al. Procalcitonin as a marker of Candida species detection by blood culture and polymerase chain reaction in septic patients. BMC Anesthesiol 2014;14:9.

25 Freydiere AM, Guinet R, Boiron P. Yeast identification in the clinical microbiology laboratory: phenotypical methods. Med Mycol 2001;39:9-33.

26 Milici ME, Maida CM, Spreghini E, et al. Comparison between disk diffusion and microdilution methods for determining susceptibility of clinical fungal isolates to caspofungin. J Clin Microbiol 2007:45:3529-33.

27 Calitri C, Denina M, Scolfaro C, et al. Etiological diagnosis of bloodstream infections through a multiplex real-time polymerase chain reaction test in pediatric patients: a case series from a tertiary Italian hospital. Infect Dis (Lond) 2015:47:73-9.

28 Dark P, Wilson C, Blackwood B, et al. Accuracy of LightCycler(R) SeptiFast for the detection and identification of pathogens in the blood of patients with suspected sepsis: a systematic review protocol. BMJ Open 2012;2:e000392.
29 Gosiewski T, Flis A, Sroka A, et al. Comparison of nested, multiplex, qPCR; FISH; SeptiFast and blood culture methods in detection and identification of bacteria and fungi in blood of patients with sepsis. BMC Microbiol 2014;14:313.

30 Markota A, Seme K, Golle A, et al. SeptiFast real-time PCR for detection of bloodborne pathogens in patients with severe sepsis or septic shock. Coll Antropol 2014:38:829-33

31 Ortiz Ibarra J, Trevino Valdez P, Valenzuela Mendez E, et al. Evaluation of the Light-Cycler $\circledast^{\circ}$ SeptiFast Test in Newborns With Suspicion of Nosocomial Sepsis. Iran J Pediatr 2015;25:e253.

32 Suberviola B, Márquez-López A, Castellanos-Ortega A, et al. Microbiological diagnosis of sepsis: polymerase chain reaction system versus blood cultures. Am J Crit Care 2016;25:68-75.

33 Grosso MJ, Frangiamore SJ, Ricchetti ET, et al. Sensitivity of frozen section histology for identifying Propionibacterium acnes infections in revision shoulder arthroplasty. J Bone Joint Surg Am 2014;96:442-7.

34 Mansat P. Frozen section histology: a new tool to identify propionibacterium acnes infections in revision shoulder arthroplasty. Commentary on an article by Matthew J. Grosso, BS, et al., "Sensitivity of frozen section histology for identifying Propionibacterium acnes infections in revision shoulder arthroplasty". J Bone Joint Surg Am 2014;96:e49.

35 Yang ZT, Wu L, Liu XY, et al. Epidemiology, species distribution and outcome of nosocomial Candida spp. bloodstream infection in Shanghai. BMC Infect Dis 2014; 14:241.

36 Cornely OA, Gachot B, Akan H, et al. Epidemiology and outcome of fungemia in a cancer Cohort of the Infectious Diseases Group (IDG) of the European Organization for Research and Treatment of Cancer (EORTC 65031). Clin Infect Dis 2015;61:324-31

37 Mansueto P, Pisciotta G, Tomasello G, et al. Malignant tumor-like gastric lesion due to Candida albicans in a diabetic patient treated with cyclosporin: a case report and review of the literature. Clin Exp Med 2012;12:201-5.

38 Ballal M, Chakraborty R, Bhandary S, et al. Candida tropicalis in a case of cholangiocarcinoma with cholangitis at a tertiary care hospital in Manipal. Med Mycol Case Rep 2013;2:95-7.

39 Lenz $\mathrm{P}$, Eckelskemper $\mathrm{F}$, Erichsen $\mathrm{T}$, et al. Prospective observational multicenter study to define a diagnostic algorithm for biliary candidiasis. World I Gastroenterol 2014;20:12260-8.

40 Jung DS, Farmakiotis D, Jiang Y, et al. Uncommon Candida species fungemia among cancer patients, Houston, Texas, USA. Emerging Infect Dis 2015:21:1942-50.

41 Li D, Li X, Xia R, et al. Molecular surveillance of candidemia due to Candida albicans among cancer patients during 2009 to 2013 by microsatellite typing. Microb Pathog 2015;81:28-32.

42 Farmakiotis D, Kyvernitakis A, Tarrand JJ, et al. Early initiation of appropriate treatment is associated with increased survival in cancer patients with Candida glabrata fungaemia: a potential benefit from infectious disease consultation. Clin Microbiol Infect 2015;21:79-86. 